Conclusion Obturator nerve injuries are a well-recognized complication of pelvic lymphadenectomy. Immediate laparoscopic nerve repair, can facilitate earlier motor recovery and prevent the need for laparotomy.

\section{SF015/\#465 PELVIC LYMPHADENECTOMY MADE EASY - A STEPWISE TECHNIQUE FOR SYSTEMATIC PELVIC LYMPH NODE DISSECTION}

S Smyth*, C Pappa, H Jiang, M Alazzam. Oxford University Hospitals NHS Foundation Trust, Gynaecological Oncology, Oxford, UK

10.1136/ijgc-2021-IGCS.59

Introduction Systematic pelvic lymph node dissection is a standard procedure conducted in the management of cervical and high-risk endometrial cancers. This requires a precise understanding of anatomical landmarks in addition to surgical approach, with present's confusion for trainees amidst multiple learning techniques. Trainees must develop economy of movement and maximise lymph node yield whilst avoiding potential complications including injury to nerves and vessels as well as post-operative morbidity.

Description Identify the boundaries - Lateral border - develop retroperitoneal space by division of round ligament laterally, divide peritoneum along the medial border of psoas muscle with identification of genitofemoral nerve toward paracolic gutter - Medial border - obliterated umbilical artery - dissect along lateral border to level of common iliac vessels bifurcation - Inferior border - obturator nerve - identify obturator foramen following pubic bone with nerve seen below - Caudal border - deep circumflex vessels - Cranial border - bifurcation of common iliac vessels - Lymph node en-bloc dissection technique - Orientation from lateral to medial and caudal to cranial - Initiate from lateral and caudal borders and dissect from external iliac vessels - Once below external iliac vein follow pubic bone to identify obturator nerve as it crosses the obturator foramen, acknowledging corona mortis vessel - Collect lymph nodes within anatomical boundaries - Ensure haemostasis.

Conclusion/Implications We present this case as an aide memoire of a basic gynaecological oncology technique. This is an essential surgical skill to develop with knowledge and practice for all subspecialists in training. https://www.dropbox.com/s/ b2k20eph9aomb5a/BPLND\%20IGCS.mp4?dl=0

\section{SF016/\#501 TECHNIQUE OF ROBOTIC ASSISTED RADICAL HYSTERECTOMY WITH BILATERAL PELVIC LYMPH NODE DISSECTION FOR EARLY CERVICAL CANCER}

J Thingujam*, R Ranade, S Dhevi RS. Narayana Mazumdar Shaw Medical Centre, Gynecological Oncology, Bangalore, India

\subsection{6/ijgc-2021-IGCS.60}

Introduction Cervical cancer is the $2^{\text {nd }}$ most common cancer in Indian women (as per globocan 2018). For many years, radical hysterectomy is the treatment of choice for early stage cervical cancer. This procedure has been traditionally performed via laparotomy but with the introduction of robotic assisted radical hysterectomy, blood loss during procedure, hospital stay, post-operative complications is relatively reduced.
Description The video illustrates a sequential narrative of operative steps of our robotic assisted radical hysterectomy and bilateral pelvic lymph node dissections in a 46-year-old female with squamous cell carcinoma of cervix. She presented with proliferative growth of $2 \times 2 \mathrm{~cm}$ in the cervix, fornices free, bilateral parametrium and rectal mucosa free. The urinary bladder was dissected downward, the retroperitoneal space opened and visualized important structures like ureters and iliac vessels. Uterine arteries cauterized, clipped and cut at the level of its origin, ureters dissected from medial leaf of broad ligament peritoneum down to its entrance into the parametrium tunnel of Wertheim. Pararectal and paravesical space created. The infundibulopelvic ligament cauterized and cut laterally, pelvic wall peritoneum of broad ligament incised downward to base of uterosacral ligament, uterosacral ligament cauterized and cut closed to rectum. Colpotomy done specimen delivered vaginally without spillage. Bilateral pelvic lymph node dissection was done and specimen delivered vaginally. vault closure done with v- lock sutures.

Conclusion Certain studies demonstrate the safety and feasibility of Robotic assisted radical hysterectomy for early-stage cervical cancer. It provides benefits such as less bleeding, reduced in hospital stay and decrease in post-operative complication

\section{SF017/\#513 PRESERVATION OF LEFT TRIPLE RENAL ARTERY DURING LAPAROSCOPIC PARA-AORTIC LYMPHADENECTOMY}

JM Eom, JS Choi*, YK Kim. Hanyang College of Medicine, Obstetrics and Gynecology, Wangsimni-ro, Seongdong-gu, Seoul, Korea, Republic of

\subsection{6/ijgc-2021-IGCS.61}

Objective To demonstrate of preservation of left triple renal artery during laparoscopic para-aortic lymphadenectomy.

Design Case study.

Settings University hospital in Korea. Patients: A 54-year-old Korean woman with postmenopausal bleeding and thickened endometrium $>3 \mathrm{~cm}$ presented to our department. The histopathology of biopsied endometrium revealed grade 2 endometrioid adenocarcinoma. The preoperative MRI shows an about $6 \mathrm{~cm}$ sized large volume of tumor within the endometrial cavity.

Interventions We perform the laparoscopic staging surgery. Firstly we performed peritoneal washing cytology, LAVH, BSO, pelvic lymphadenectomy. We designated four area for para-aortic lymphadenectomy. During the procedure in area 4, it was confirmed that two left renal arteries were derived from the trunk of the aorta below the left renal vein. The left lower segmental artery was derived from the middle part of the inferior mesenteric artery and left renal vein. The middle segmental artery was derived just below left renal vein. The left main renal artery was located on the dorsal side of the left renal vein at its normal position. We carefully resected the para-aortic lymph nodes to prevent variant renal artery damage.

Conclusions Laparoscopy is a feasible and safe approach to diagnosis of vascular anomaly during para-aortic lymphadenectomy for gynecologic malignancies. It is very important for the gynecologic oncologist to have knowledge of retroperitoneal vascular anatomy, experience in laparoscopic surgery, and an accurate surgical technique to avoid vascular injury during laparoscopic para-aortic lymphadenectomy. 